\title{
Téoros
}

Revue de recherche en tourisme

\section{Grandeurs et déclin du centre d'interprétation}

\section{Luc Noppen et Lucie K. Morisset}

Volume 24, numéro 2, été 2005

URI : https://id.erudit.org/iderudit/1071092ar

DOI : https://doi.org/10.7202/1071092ar

Aller au sommaire du numéro

\section{Éditeur(s)}

Université du Québec à Montréal

\section{ISSN}

0712-8657 (imprimé)

1923-2705 (numérique)

Découvrir la revue

\section{Citer cette note}

Noppen, L. \& Morisset, L. K. (2005). Grandeurs et déclin du centre

d'interprétation. Téoros, 24(2), 72-75. https://doi.org/10.7202/1071092ar d'utilisation que vous pouvez consulter en ligne.

https://apropos.erudit.org/fr/usagers/politique-dutilisation/ 


\section{Grandeurs et déclin du centre d'interprétation}

\section{Luc Noppen et Lucie K. Morisset}

Les centres d'interprétation semblent avoir la cote ces jours-ci. Une simple recherche «Google» de «centre d'interprétation » renvoie plus de 42000 pages. La Société des musées québécois liste 238 «lieux d'interprétation » au Québec, soit un pour 30000 habitants ou un pour 745 touristes «internationaux » entrés au Québec en avril 2005, sans compter les musées et autres centres d'exposition qui ne sont pas moins nombreux. Le registraire des entreprises du Québec, pour sa part, dénombre 114 «centre[s] d'interprétation »: de la framboise (Saint-Pacôme); du granit (Stanstead); des fossiles (Île-d'Anticosti); de l'eau (Laval); de l'érable (Saint-Prosper, et de l'érablière à Saint-Ubalde et à Lachute); des baleines (Percé); du bleuet (Saint-Bruno); des anges (Sainte-Anne-de-Beaupré); de la vie urbaine (Québec); des Irlandais (Charlesbourg, Québec) ou encore, évidemment, du patrimoine (Plaisance). Cent quatorze, c'est deux fois le nombre d'entreprises ainsi dénommées qui existaient au Québec avant 1994. J umeler ce corpus à celui des quelque 300 entreprises québécoises dont la mission a trait au «patrimoine » dope l'imagination.

Au même moment où Amandine, cette internaute d'un blogue intitulé J ournal de mon séjour au Québec, signale, "c'est très beau [le Québec] mais un peu trop touristique ${ }^{1} \gg$, d'où vient, alors, ce sentiment de déception de certains qui, déplorant l'homogénéité, l'absence de surprises ou le peu de spectaculaire du paysage touristique du Québec, vont jusqu'à dire qu'ils préféreraient de loin découvrir la ressource brute, en aval de ses appareils de mise en valeur et d'interprétation?

Constatons d'abord que la recherche «Google » en français sur «centre d'interprétation » renvoie près de $30 \%$ plus de résultats que son équivalent en anglais ${ }^{2}$; de fait, la majorité des «centres d'interprétation » de cette planète ont pignon sur rue au Québec. Ce phénomène, qui ne peut s'expliquer que partiellement par la nécessité pour les musées (et non pour les centres d'interprétation) d'obtenir une accréditation de leur mission de consenvation et des conditions qu'ils offrent à cet égard, ne réduit pas nécessairement la valeur des centres d'interprétation, mais révèle cependant l'utilité qu'ils ont eue, au moins à un moment de leur histoire. Ainsi, la Commission des monuments historiques du Québec, à défaut de connaître un moyen de valoriser le site de la bataille du Long-Sault - où Dollard des Ormeaux est réputé avoir, en 1660, sauvé Montréal des mains des Iroquois -, relégua ce projet de commémoration aux oubliettes.
Le site fut englouti lors de la construction du barrage de Carillon en 1962, à peine six ans après que la bataille eut été désignée «événement historique national » par le gouvernement fédéral. II n'y a guère plus aujourd'hui, pour en rappeler la mémoire, que le monument aux «héros » de la bataille qu'on avait élevé non loin en 1919. C'est que, lors de l'inondation du site, et avant, quand l'événement interpellait la Commission des monuments historiques, on ne connaissait pas le désormais populaire «centre d'interprétation » qui aurait densifié l'intérêt des lieux.

L'historien du patrimoine Martin Drouin a déjà écrit l'histoire du centre d'interprétation québéco is et fait la démonstration de son originalité (Drouin, 2002 : 23-31). Nous retracerons donc plutôt ici les grandes lignes de ce qui a bien pu lui advenir, en amont et en aval de son épanouissement.

\section{De l'interprétation au centre d'interprétation}

Bien avant que des centres d'interprétation, comme celui qui aurait pu pérenniser la bataille du Long-Sault, permettent de rappeler aux sens ce qu'il n'était plus possible de voir, l'interprétation, ou plutôt la compréhension, était le fait d'une culture commune et préalable, de connaissances partagées, par exemple par ces «connoisseurs » ou par les aristocrates britanniques qui complétaient leur éducation dans la contemplation des ruines vers lesquelles les conduisait le Grand Tour. Qui visitait un site ou un monument, bref, était a priori réputé en mesure de s'en représenter les significations et l'intérêt. Dans ce contexte, l'idée d'interprétation est née dans la médiation entre un paysage et un public, ou entre quelque collectionnement et ses «connoisseurs » auxquels un individu, généralement érudit, proposait le sens qu'il avait décodé, d'un objet ou d'un lieu. L'interprétation proprement dite apparut de la volonté libérale de démocratiser l'art et l'histoire, «si bons pour le peuple»: «in our modern complex world», comme on l'écrivit alors, «the formal education of youth in schools and colleges is not enough. Neither is the more or less formal education of adults [sufficient] to meet the great need » (C rittenden, 1977). C'est donc en vue, d'abord, de faire partager à un plus grand nombre, qu'on inventa le conférencier des monuments historiques, puis le guide-interprète, en croyant pouvoir faire l'économie d'une culture générale, voire éventuellement spécialisée en arts, en ethnologie, en archéologie, etc.

On réalisa néanmoins bientôt que ces interprétations étaient trop rattachées, évidemment, à la personnalité de l'interprète dont on convoquait les connaissances; certains sites, du fait de discours de 


\section{sonet}

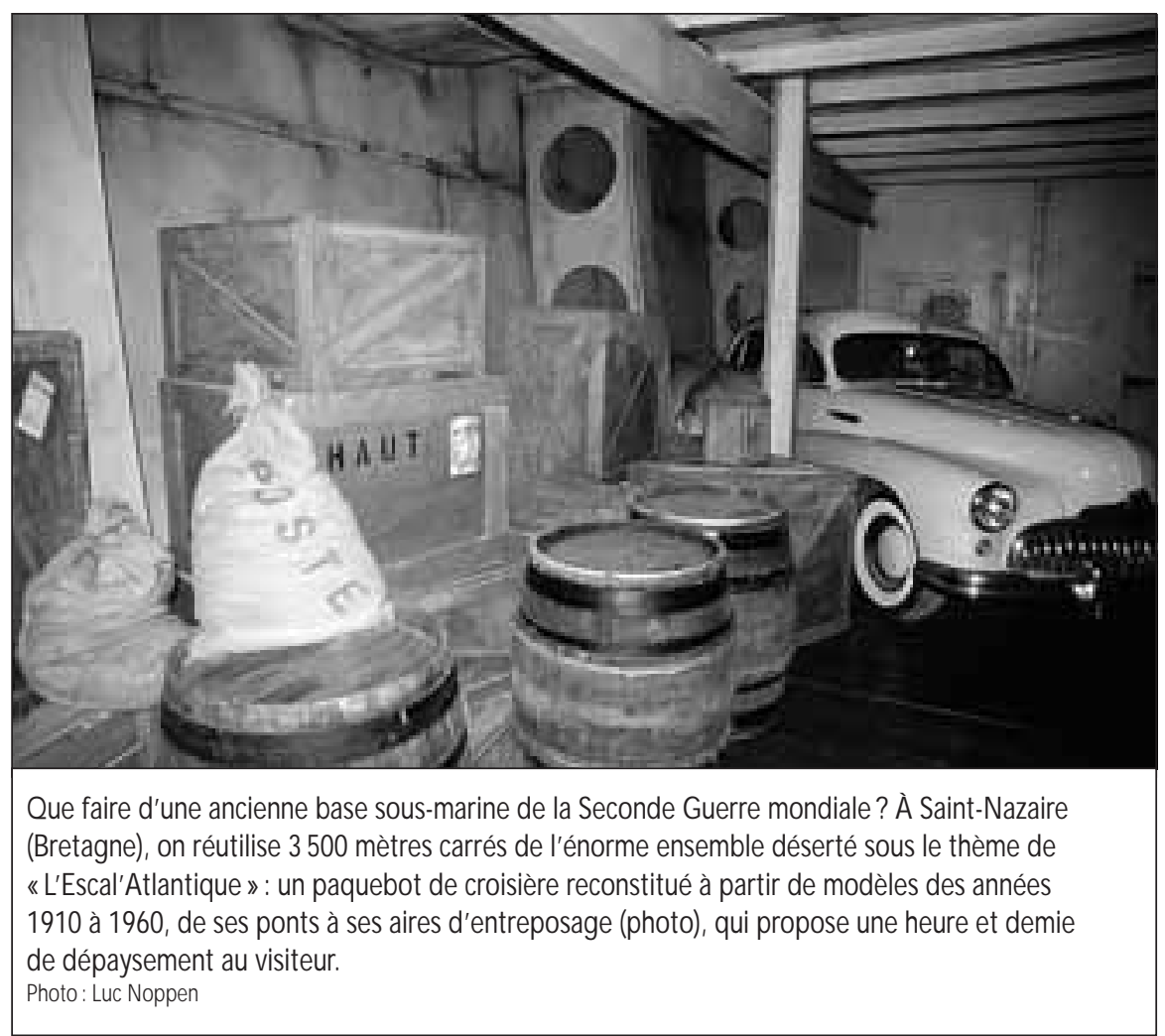

En anglais, aujourd'hui, interpretation connaît un cas d'espèce répertorié et lexicalisé : le heritage interpretation. Comme en français, on renvoie maintenant à «l'action d'expliquer, de donner une signification claire (à une chose obscure) ou son résultat ». L'Office québécois de la langue française est plus explicite encore; logée à l'enseigne de l'industrie touristique, «l'interprétation » consiste à «transmettre de l'information aux clients, de manière à leur faire comprendre, apprécier et respecter divers éléments du patrimoine naturel et culturel ». L'organisme va jusqu'à codifier le «centre d'interprétation » comme le «bâtiment principal d'un milieu patrimonial où sont regroupées diverses activités liées à l'interp rétation et où les visiteurs peuvent habituellement obtenir des renseignements sur les services connexes offerts », sachant que le centre d'interprétation «peut renfermer des bureaux, une salle d'exposition, une salle de projection, des salles de repos, un casse-croûte, une boutique de cadeaux, etc. »

Ainsi, le véritable mouvement lancé en 1957 par l'ouvrage de Tilden, Interpreting Our Heritage, ne s'est pas ralenti depuis, bien au contraire. Les moyens déployés sont certes moins nouveaux qu'encore dix ans plus tard, quand on annonçait «this new channel, this new mean of reaching our

constructions nationales, pouvaient ainsi se révéler sous un jour trop politique ; d'autres, en raison de l'érudition de l'interprète, continuaient de paraître abscons. L'institutionnalisation à peu près simultanée du patrimoine, des musées et de la culture demandait un contrôle des contenus : «most of our museums », écrivit-on par la suite de cette «émancipation», «were stuffy and lacking in popular appeal, mere masses of ill-assorted oddities that had been brought in because someone happened to take a fancy to them » (Crittenden, 1977 : XV). Bien curieusement, alors, on adopta les dispositifs muséologiques comme mécanismes d'interprétation : le centre d'interprétation était né. Il s'agissait, alors qu'on se refusait à interpréter les œuvres d'art in situ dans les musées, de créer d'autres musées extérieurs à l'objet ou au lieu interprété, mais qui - on devrait bien le réaliser un jour -, en reproduisant en quelque sorte l'objet interprété (dans un panorama, dans des maquettes ou autres exhibits) pour produire du sens, désaffectaient l'authentique, à tout le moins l'objet même de l'interprétation.

Avec l'éclatement de cette culture commune de jadis et avec l'intensification, encore plus manifeste, de l'exploitation touristique à des fins commerciales, on en était arrivé à définir l'interprétation comme science. En 1957, le père de cette «discipline », Freeman Tilden (1957 : 3), écrivait encore :

The word interpretation as used in this book refers to a public service that has so recently come into our cultural world that a resort to the dictionary for a competent definition is fruitless. Besides a few obsolete meanings, the word has several special implications still in common use: the translation from one language into another by a qualified linguist; the construction placed upon a legal document; even the mystical explanation of dreams and omens. people, through their parks, their museums, and their historic sites. [... ] This new opportunity lies in the realm of what has come to be called 'interpretation.' » (C rittenden, 1977 : XV) Les parcs, les musées et les sites historiques ne sont plus les seuls sujets de l'interprétation, dans ces «attractions en soi » que se veulent de plus en plus de centres d'interprétation; ce phénomène d'expansion, qu'il faut alors mettre en lien avec la mondialisation de l'administration du patrimoine représentée par l'Unesco et avec l'émergence du «patrimoine immatériel » qui s'en est suivie, veut en effet que l'on puisse dorénavant interpréter le visible comme l'invisible, et le réel comme l'impossible.

On est allé très loin dans l'apposition de pages de textes sur des murs et dans la réplique d'artefacts qui pouvaient être visualisés, manipulés, brisés et reconstruits, ce que n'autorisait pas l'authentique. Pourtant, si les préfaciers de Tilden ne pouvaient imaginer que l'on interprète autre chose que ces musées, parcs et sites historiques qu'ils administraient, ils avançaient cette mise en garde, empruntée à Anatole France: «Do not try to satisfy your vanity by teaching a great many things. » (Hartzog, 1977 : XIII) C'est néanmoins ce qu'on a fait, puisque cette multiplication s'imposa dès lors que l'interprétation fut aussi mise en charge de créer le contenu. Le centre d'interprétation amorçait son déclin le jour de sa naissance même.

Il eut, certes, une période de grandeur, au Québec à tout le moins, où l'architecture de quelques centres d'interprétation, dont les remarquables Bourg de Pabos et Parc de l'aventure basque (Trois-P istoles), a permis à de jeunes architectes de faire leurs dents sous les auspices des budgets culturels québécois, moins importants bien sûr que ceux qui ont permis, à d'autres et ailleurs, de signer les notoires musée Guggenheim de Bilbao ou Musée de l'histoire juive de Berlin. Mais même cette phase, qui a tout de même livré au paysage québécois 
quelques hauts lieux d'une modernité reconquise, semble plutôt derrière que devant, et pas qu'au Québec : ceux qui fréquentent les États-Unis connaissent les mésaventures du Cyclorama de la bataille de Gettysburg, œuvre importante de l'architecte Richard Neutra, construite en 1961 et menacée depuis 1996 de remplacement par un centre d'interprétation plus «performant».

C'est qu'en confiant à l'interprétation de définir le contenu, on devait en arriver au point où, comme le redoutait Marshall McLuhan, "The medium is the message " $^{3}$ et de là découvrir, au bout de quelques années d'expériences, que l'interprétation, quel que soit le site, était toujours semblable, du fait d'une typologie développée par un corps de professionnels (graphistes, muséologues, etc.) grandissant qui ne concernait guère, ni ne prit en compte, la distinction ou la différence des lieux ou des objets. Du site qu'on devait interpréter pour le voir, la logique, pendant ce temps, s'est renversée et il est peu de tours d'observation ou de fortifications qui ne soient pas dotées (à côté), de l'un de ces centres d'interprétation qui ont au moins pour intérêt de créer de l'emploi, pour des guides-interprètes, certes, mais encore plus pour tous ces «spécialistes de l'interprétation » et autres «firmes et travailleurs spécialisés en muséologie et en patrimoine », comme les recense la Société des musées québécois. Ceux-là, substitués au conservateur d'antan, font maintenant leur pain quotidien du patrimoine comme de la mise en tourisme, sous prétexte, entre autres, de «prolonger le séjour du visiteur».

Or, la lassitude - nous y revenons plus loin - provoquée par des centres d'interprétation qui, en dépit des millions investis, finissent par se ressembler davantage que se distinguer, survient en parallèle de l'éclosion du virtuel. On a d'abord, bien sûr, utilisé cet épanouissement de la technologie dans les centres d'interprétation, dotés de «multimédias » de plus en plus fastes. Mais, en définitive, si le trai- tement virtuel - le 3D, par exemple - permet d'accéder aux significations des sites beaucoup plus efficacement, pourquoi se déplacerait-on vers un centre d'interprétation alors qu'on peut convoquer l'interprétation chez soi? Et après, seulement, aller voir le lieu ou l'objet, c'est-à-dire redevenir cet homme cultivé qui faisait le Grand Tour.

Comme le virtuel s'est substitué, en tant que mode de connaissance, au texte historique ou au livre qui permettait, à une époque, d'accéder à l'objet d'art, on pourrait donc maintenant s'attendre à ce que disparaissent les artefacts d'interprétation dont on a affublé les musées d'art et tous ces panneaux qui jonchent les lieux d'intérêt et leur proximité. Le centre d'interprétation ne sera plus qu'un vestige de l'autre époque, celle qui est révolue, qui a d'abord permis d'interpréter les hauts lieux, les grands événements, les phénomènes d'envergure et qui s'est échouée dans le banal. Ceux qui, méfiants (ou sans moyens financiers) face à la grande vogue du multimédia et du virtuel, ont exploré, beaucoup plus que l'interprétation, des formes d'animation qui permettaient de comprendre les usages et les coutumes, peuvent le réaliser aujourd'hui : il est beaucoup plus attachant de faire l'expérience de l'animation d'un site que d'être assujetti à des codifications dévolues à un public-cible d'un âge moyen qui bien sûr, comme toutes les moyennes, n'existe pas. En revanche, le visuel qui domine l'univers du virtuel ne peut satisfaire à l'expérience, du timbre de voix qui transmet une chanson, du savoir-faire qui fabrique un vêtement; nous voilà donc de retour à la qualité de l'interprète (qui dépend de l'apparence d'authenticité des personnages, non de la justesse de leur message) et, d'une certaine façon, à la définition véritable que McLuhan (1964) donnait au médium : "any extension of ourselves ». "Of ourselves», en effet, plutôt que d'une distante institution patrimoniale, d'une soi-disant rentable industrie touristique ou de cette multitude de firmes spécialisées dans «l'interprétation».

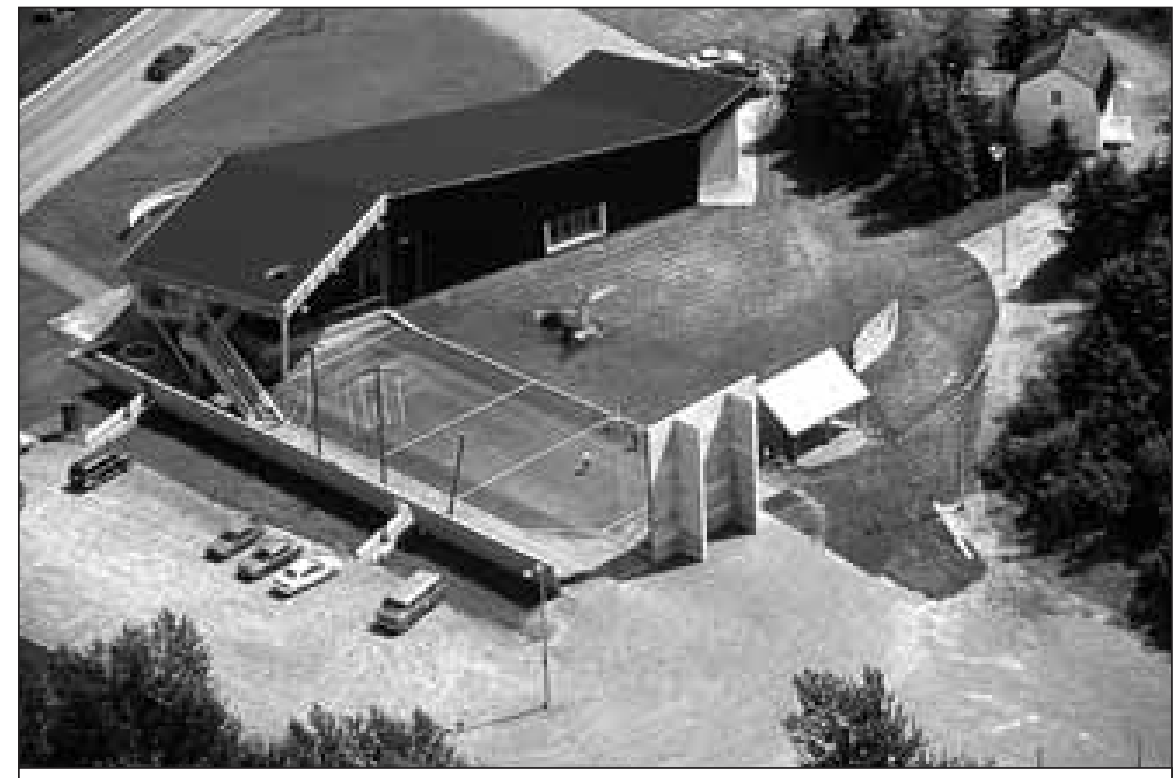

À Trois-Pistoles, le Parc de l'aventure basque en Amérique, œuvre des architectes Cormier, Cohen, Davies (1996), regroupe un centre d'interprétation voué à «faire revivre » I'histoire des aventuriers basques, un café et une place publique, réputée "seul fronton de pelote basque au Canada », qui accueille annuellement un tournoi international de pelote basque.

Photo : Pierre Lahoud
Si, dans cet état actuel des choses, les centres d'interprétation devaient être promis à quelque avenir, par exemple parce que le touriste du Grand Tour, en dépit de la virtualisation des connaissances, ne reviendrait pas, il faudra tout de même, espérons-nous, tenter d'en contrer deux effets pervers. Le premier tient de l'institutionnalisation de l'ignorance ; McLuhan soulignait lui-même la fatalité qui guettait le surdéveloppement de tout «medium», par exemple de l'automobile de favoriser l'atrophie des muscles et, dans tous les cas, d'aller à l'encontre des bénéfices originellement escomptés. Bien que des «formations en interprétation du patrimoine » existent désormais - celles offertes par l'Association québécoise d'interprétation du patrimoine ne requièrent que quinze heures, au coût de 3000 \$, dont jusqu'à $75 \%$, nous apprend l'association, peut être financé dans le cadre des partenariats avec les Conseils régionaux de la culture et les Centres locaux d'emplois régionaux ${ }^{4}$-, il faudra bien admettre que, sous le patrimoine et plus encore sous l'interprétation, le lieu et l'objet qui motiveraient le site touristique sont des objets de science et de compétence, comme la mécanique des fluides 
ou l'anatomie ; l'obligation, faite par l'urgence de la mise en tourisme et la rentabilité de ses industries, de se replier éternellement sur des savoirs de plus en plus anciens ne conduit qu'à générer, comme une machine emballerait des aliments préparés, des interprétations forcément de plus en plus simplistes peu propices à intéresser des citoyens par ailleurs de plus en plus informés.

Le second effet pervers, ancré dans la définition du centre d'interprétation, tient de l'itinéraire du touriste, qui bien souvent en fera le tour pour en repartir sans même considérer ce que le centre d'interprétation (du site de bataille, du village, de la pomme de terre ou du granit de Stanstead) devait donner à voir. Cela surviendra d'autant plus si le touriste en est à son huitième centre d'interprétation (cela, bien sûr, relève aussi de la multiplication, sous différents prétextes qui pourraient faire l'objet d'une autre chronique, des «sites touristiques », qui est loin d'être proportionnelle au nombre de touristes qui pourraient les fréquenter). L'énigme n'est pourtant pas bien compliquée; I'industrie touristique est au demeurant bien familière avec cette équation, codifiée par J oe P ine, selon laquelle l'efficacité de l'expérience touristique se mesure au produit, divisé par les coûts d'accès, du nombre de touristes concernés, du temps consacré à l'attraction, de l'attention qui lui est portée, de l'intensité de l'expérience et de sa mémorabilité (Pine et Gilmore, 1999). Or, puisque la durée de l'expérience n'augmentera jamais suffisamment pour compenser la diminution de l'attention (diluée entre les 114 «centres d'interprétation » par exemple), de l'intensité (nous avons évoqué combien, l'institutionnalisation des professions concernées aidant, les centres d'interprétation brillent pour la plupart de la même intensité) et de la mémorabilité («c'était où, donc?»). Ce phénomène, qui nous ramène à la lassitude décrite précédemment, a ainsi pour conséquence le second effet pervers que nous énoncions, qui n'est lui-même pas sans conséquence. Car il y a fort à parier que, de centre d'interprétation en centre d'interprétation dans lesquels le touriste, s'il veut en voir quelques-uns, reste confiné, l'expérience du centre d'interprétation se substituera à celle de la ressource. Dans le cas d'une ressource tangible (une maison, un village, une rivière), cela serait bien ennuyeux, à tout le moins dans la perspective où la mise en tourisme fut à un moment considérée par ses promoteurs comme un moyen de soutenir (culturellement, économiquement) la conservation. À terme, on pourrait donc raser les paysages et se débarrasser de toutes ces choses que les centres d'interprétation interprètent pour ne conserver, au bout du compte, que les centres d'interprétation. Enfin, on aurait au moins réglé le problème de l'entretien du patrimoine.

Historiens d'architecture, Luc Noppen, titulaire de la Chaire de recherche du Canada en patrimoine urbain, et Lucie K. Morisset sont professeurs au Département d'études urbaines et touristiques de I'Université du Québec à Montréal et chercheurs au Centre interuniversitaire d'études sur les lettres, les arts et les traditions.

Notes

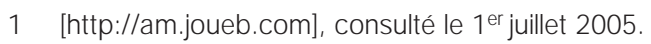

2 Il convient d'exclure à ce titre, évidemment, les quelque 30 millions de pages d'Internet sur des visitor centres, ancêtres à la fois des kiosques d'information touristique et des centres d'interprétation...

3 Qui voulait non pas annoncer que le contenant devrait surpasser le contenu, mais observer la superficialité des perceptions.

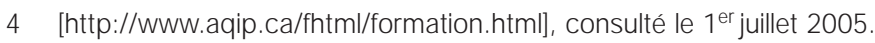

\section{Bibliographie}

Crittenden, Christopher (1977), "Preface to the Second Edition", dans Freeman Tilden (dir.), Interpreting Our Heritage, University of North Carolina Press.

Drouin, Martin (2002), «Le centre d'interprétation: un produit touristique incontournable né d'une révolution muséale», Téoros, vol. 21, n² 2, p. 23-31.

Hartzog, B. George (1977), "Foreword to the Second Edition", dans Freeman Tilden (dir.), Interpreting Our Heritage, University of North Carolina Press.

McLuhan, Marshall (1964), Understanding Media: The Extensions of Man, New York, McGraw Hill.

Pine, B. J oseph, et J ames H. Gilmore (1999), The Experience Economy: Work Is Theatre \& Every Business a Stage, Boston, Massachusetts, Harvard Business School Press.

Tilden, Freeman (1957), Interpreting Our Heritage, University of North Carolina Press. 